\title{
Estudio de materiales y técnica de ejecución de los restos de pintura mural romana hallados en una excavación arqueológica en Guadix (Granada)
}

\author{
Ana García Bueno *, Andrés M. a Adroher AurouX ** ${ }^{*}$ M. ${ }^{\text {a }}$ Carmen López \\ PertíñeZ ${ }^{\star \star *}$, Victor J. MEdina Flórez ${ }^{\star \star \star *}$
}

\begin{abstract}
RESUMEN
ABSTRACT

Este artículo da a conocer unos restos

This article makes Known the de pintura mural romana encontrados en Guadix, tanto desde el punto de vista arqueológico como analítico.

Estos fragmentos de pintura mural constituian parte del material de relleno de una canalización romana, cuya cronología pudo precisarse con exactitud, que posiblemente formaria parte de la red hidráulica de la antigua

ACCIS. Ateniéndonos a las características de los fragmentos, se seleccionaron muestras de los colores más frecuentes para determinar los pigmentos utilizados y la técnica de ejecución empleada en su construcción. remains of Roman wall painting found in Guadix, both fron au archaeological and analytical point of view. These fragments of wall painting constituted part of the filling material used in a Roman channelling, whose chronology was accurately established, which would probably be part of the hydraulic system in ancient ACCIS. Keeping in mind the characteristics of the fragments, samples of the most frecuent colours were chosen to determine the pigments used and the execution technique employed in its construction.
\end{abstract}

\footnotetext{
* Departamento de Pintura de la Facultad de Bellas Artes de Granada (Universidad de Granada).

** Departamento de Prehistoria y Arqueología. Facultad de Filosofía y Letras de Granada (Universidad de Granada).

** Instituto «Gómez-Moreno" de la Fundación Rodríguez-Acosta.

*** Departamento de Pintura de la Facultad de Bellas Artes de Granada (Universidad de
} Granada). 


$\begin{aligned} \text { PALABRAS CLAVE } & \text { KEY WORDS } \\ \text { Pinturas, murales, pigmentos } & \text { Paintings, murals, natural pigments, } \\ \text { naturales, pigmentos artificiales, } & \text { artificial pigments, agglutinats, } \\ \text { aglutinantes, morteros. } & \text { mortars. }\end{aligned}$

\section{INTRODUCCIÓN}

Los fragmentos de pintura mural estudiados en este trabajo proceden de la excavación arqueológica llevada a cabo en la casa $n .{ }^{\circ} 3$ de la calle Santa M. ${ }^{a}$ del Buen Aire, en Guadix (Granada). Se seleccionaron por presentar una gran diversidad de materiales, sobre todo pigmentos, y unas características de textura y brillo que nos hicieron pensar en una técnica especialmente cuidada, por lo que nos pareció interesante hacer un estudio de materiales completo, que permitiera, en la medida de lo posible dadas las pequeñas dimensiones de los fragmentos, definir la técnica de ejecución empleada para la realización de los mismos ${ }^{1}$.

Puesto que resulta imposible determinar la técnica de una pintura si no se conoce el material que fija los pigmentos, sea éste orgánico o inorgánico, es imprescindible detectar e identificar si se empleó materia orgánica como aglutinante de la capa pictórica y en caso de haberlo cuál sería el empleado.

Abad Casal (1982: 126) en su obra sobre la pintura mural romana en España, hace la siguiente alusión sobre unas pinturas murales romanas encontradas en Guadix: "1.1: VILLA PAULENCA; en el sector A-5 aparecieron treinta fragmentos de revestimientos pintados, posiblemente del sector residencial 5, aún sin excavar. El color más frecuente era el rojo; franjas rojas, azules y negras estaban separadas por bandas de color marfil o hueso de $3 \mathrm{~cm}$. de ancho. Aparecieron asi mismo otros fragmentos de color castaño oscuro». Esta noticia nos sirve como referencia más próxima al confirmar la existencia de restos similares en la zona.

Normalmente los fragmentos de pintura mural de este tipo, procedentes de excavaciones y de carácter exento, no son considerados hallazgos importantes ya que aparecen desprendidos del paramento sobre el que se crearon y generalmente presentan graves problemas de conservación, por lo que son pocos los estudiados. Sin embargo pueden

1 Excavaciones de urgencia en Guadix (Granada), año 1994; coodirigidas por los arqueólogos Dr. Cristobal Gonzalez Roman (Dpto. Historia Antigua, Granada) y Dr. Andrés Adroher Auroux (Dpto. de Prehistoria y Arqueologia, Granada). 
llegar a ser tan valiosos como cualquier otro material arqueológico. La determinación de sus características técnicas y formales contribuye a su datación y consecuentemente a la reconstrucción histórica de una secuencia arqueológica, pudiéndose llegar a concretar a través de ellos las características de las viviendas, los aspectos económico-sociales, estéticos, etc. de un periodo.

Este trabajo hay que encuadrarlo dentro de la línea de investigación iniciada en el Dpto. de Pintura de la Facultad de Bellas Artes de la Universidad de Granada relativa al estudio de las técnicas de pintura mural y de su conservación y restauración.

Los resultados obtenidos aportan datos que consideramos de interés tanto en sí mismos, como en relación con los que aparecen en trabajos ya publicados o de próxima publicación que se vienen desarrollando sobre las técnicas y evolución de la pintura mural.

\section{CONTEXTO HISTÓRICO-ARQUEOLÓGICO}

El conjunto de la Unidad Geomorfológica donde actualmente se asienta el núcleo del casco urbano de Guadix, parece haber sido ocupado a partir de los primeros momentos de la Edad del Bronce, durante la primera mitad del segundo milenio a.C. Desde entonces y hasta la actualidad, la ocupación de las colinas de Guadix ha sido una constante histórica sin solución de continuidad.

El objetivo primordial de la excavación realizada en el antiguo «Hospital Real» de Guadix (lámina I), entre los meses de octubre y noviembre de 1994, consistia en la documentación de una canalización romana aparecida en el patio del edificio; ésta discurría en sentido este-oeste, en un estado de conservación realmente excelente. Por el estudio de los materiales cerámicos asociados y según nuestros conocimientos actuales, podemos situar su construcción, en el segundo cuarto del siglo I d.C.

Las muestras analizadas forman parte del conjunto de materiales arqueológicos encontrados en esta excavación de urgencia, concretamente los relacionados con la construcción y el posterior abandono de la canalización.

Los fragmentos proceden del primer nivel de relleno de la canalización (4111 y 4112) o bien de los rellenos concentrados en torno a los registros de acceso que existen desde la parte superior de la misma, y que presentan gran cantidad de materiales de construcción pertenecientes a estructuras presumiblemente domésticas (tegulae, enlucidos estucados y pintados, ladrillos, ímbrices, piedras, etc.). 


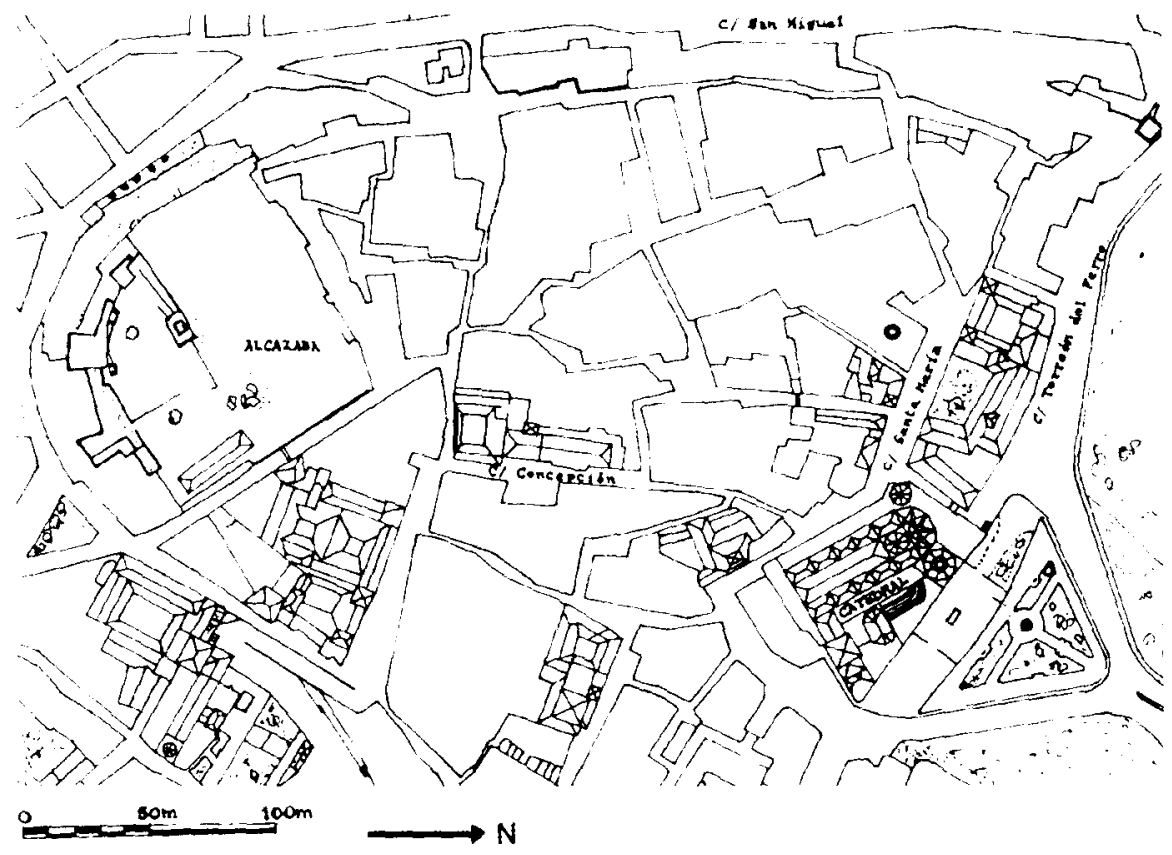

Lámina I

El contexto arqueológico se conforma por presencia de terra sigillata sudgálica, sin otras producciones, lo que nos permite definir la segunda mitad del siglo i d. C. como fecha para la formación del relleno y, correspondientemente, para la construcción de la canalización.

El nivel 4051 es el relleno para la construcción de una tubería de plomo. La cronología debería establecerse en la segunda mitad del siglo I d.C. (del año 25 al 50). Es una unidad relacionada con rellenos posteriores a la construcción de la canalización; la profundidad media es de $1,68 \mathrm{mts}$; granulométricamente está compuesta por arenas y limos muy permeables. Su cronología es muy fiable tanto por el escaso número de intrusiones detectado en el material cerámico, como por sus correspondencias estratigráficas.

El índice de humedad es muy alto, sobre todo en las zona más profundas, ya que existen afloramientos de la capa freática, a 2,10 mts. de la superficie actual, y humidifican fuertemente los materiales aparecidos. Además, en el caso de la tubería de plomo, se observó agua en su interior. 


\section{CARACTERISTICAS DE LOS MATERIALES ENCONTRADOS EN LA CANALIZACIÓN}

Los materiales encontrados en el interior de la canalización, fueron depositados allí como desecho. La canalización fue utilizada como basurero hasta quedar completamente cegada. El espacio de tiempo que tardó en inutilizarse debió ser breve, como refleja el intervalo cronológico que aporta el estudio del material cerámico de su interior (40 años de uso). Después de este acontecimiento no volvió a utilizarse y se edificó sobre la mayor parte de ella.

Como se indica en el apartado dedicado al contexto arqueológico, los fragmentos de pintura mural analizados pertenecientes a los niveles 4111 y 4112 formaban parte del relleno excavado del interior de la canalización. Las propias características de la obra, construida en opus caementicium, (por tanto, con gran cantidad de cal) así como su funcionalidad (conducción de agua), determinan la conservación de las piezas; que además estuvieron expuestas a un ambiente postdeposicional de alta humedad, proveniente de diferentes filtraciones.

Gracias a que el secado de los fragmentos fue lento, se disminuyeron los riesgos de pulverulencia, disgregaciones, etc., quedando en un relativo buen estado de conservación y manteniéndose en muchos casos la película pictórica homogénea y con aspecto satinado.

Los fragmentos de pintura mural son placas irregulares (longitud máxima $15 \mathrm{~cm}$ ), que conservan buena parte de los morteros de preparación (grosor máximo conservado $8 \mathrm{~cm}$.). Por sus pequeñas dimensiones los restos de motivos decorativos son mínimos. Estos pueden ser de carácter arquitectónico, constituidos por trazos, líneas o ángulos. Por comparación con otros ejemplos conocidos debieron ser superficies amplias, divididas en espacios cuadrados o rectangulares a modo de casetones con variada policromía de imitaciones a mármoles y probablemente con algunos motivos vegetales. El desarrollo de temas decorativos vegetales o figurativos, debía estar limitado a zonas muy concretas sin extenderse con profusión, de ahí la dificultad de encontrarlos y la imposibilidad de reconstruir ni siquiera un mínimo del esquema del dibujo. Por similitud estilística y cercanía geográfica podrían tener paralelismos con las pinturas murales del Ruedo de Almedinilla (S. I d. de C.) (Vaquerizo, 1994).

Encontramos frecuentemente superposición de líneas de colores, negro y rojo, sobre fondo blanco, o líneas blancas, negras y ocres sobre fondo rojo, imitaciones a mármoles, cambios de tonalidad roja, etc. Se puede intuir, por 
la calidad, variedad y cantidad de pigmentos, que el resultado final debía ser de gran riqueza, lo que demostraria la existencia de construcciones nobles y en consecuencia la importancia de Guadix como ciudad romana.

\section{CRITERIOS DE SELECCIÓN DE MUESTRAS. IDENTIFICACIÓN POR SIGLAS}

Teniendo en cuenta que contábamos con gran cantidad de fragmentos de pintura mural en esta excavación, y que no podiamos analizar más que a un número reducido de ellos, decidimos elegir los más significativos y los que pudieran aportar más información, tanto en este primer momento, como en los estudios que posteriormente se iban a llevar a cabo.

La elección de las muestras cuyos resultados exponemos en este articulo, estuvo basada en todo momento, en una serie de criterios selectivos:

* Por un lado la fiabilidad de la cronología de los niveles excavados, lo que nos llevó a elegir aquellos en los que las posibilidades de intrusiones fuesen mínimas. Los niveles 4111,4112 y 4051 , son niveles cerrados, con cronología muy fiable.

* Por otro lado se tuvo en cuenta el estado de conservación de los fragmentos. Como ya señalábamos uno de los mayores problemas que encontramos al estudiar materiales arqueológicos es su estado de conservación. En general suelen tener alteraciones derivadas del lugar donde estuvieron depositados (alteraciones postdeposicionales), y estas pueden afectar especialmente a los resultados estudios analíticos. Además, considerando la posibilidad del empleo de aglutinantes en la capa pictórica, se tuvo especial cuidado en que ésta presentara buena consistencia y cohesión, así como que tuviera un aspecto satinado y homogéneo, características que normalmente reflejan la buena conservación de estos componentes.

* Otro aspecto que determinó la selección fue el técnico. Es decir, se intentó conseguir abarcar toda la variedad cromática que aparecía en el conjunto de fragmentos de la excavación, de tal manera que pudieran aportar datos significativos sobre su composición y técnicas de aplicación. Asi en la Unidad Estratigráfica donde existían varios fragmentos de estuco con capa pictórica de un mismo color, se seleccionaron sólo aquéllos que presentaban restos de decoración y diferencias cromáticas, de tal manera que en el conjunto seleccionado pudiera encontrarse al menos una muestra de cada color.

La identificación de los fragmentos en la excavación se realiza por medio de la sigla que funciona como registro arqueológico, y se aplica a todos los 
materiales encontrados en la misma. Por ej. GUA-4111-E-1 significa: Guadix / Hospital Real, nivel estratigráfico 111/ pintura mural / pieza 1.

Para facilitar la identificación de las muestras y la comparación de resultados en el estudio material, se ha modificado la nomenclatura arqueológica, creándose una especifica, en la que por ej. HR-P1-Am significa: Hospital Real de Guadix/ pintura mural, número asignado a la unidad estratigráfica / inicial del color del fragmento, en este caso amarillo.

Para no perder los datos arqueológicos iniciales, ni la relación de las muestras con los fragmentos, hemos creado la siguiente tabla de equivalencias:

Tabla de equivalencias de siglas empleadas

\begin{tabular}{ll}
\hline $\begin{array}{l}\text { Siglas de } \\
\text { excavación }\end{array}$ & $\begin{array}{l}\text { Nomenclatura } \\
\text { Estudio de materiales }\end{array}$ \\
\hline GUA-4111-1 & HR-P1-Am. (Amarillo) \\
GUA-4111-4 & HR-P1-A. (Azul) \\
GUA-4111-5 & HR-P1-N (Negro) \\
GUA-4111-12 & HR-P1-R. (Rojo) \\
GUA- 4112-1 & HR-P2-R. (Rojo) \\
GUA- 4112-1 & HR-P2-V. (Verde) \\
GUA-4112-1 & HR-P2-B (Blanco) \\
GUA-4112-2 & HR-P2-R1. (Rojo) \\
GUA-4112-3 & HR-P2-R2. (Rojo) \\
GUA-4051-1 & HR-P3-A (Azul) \\
\hline
\end{tabular}

\section{METODOLOGIA}

La metodología seguida en el estudio de estas pinturas está encaminada a la determinación de su técnica de ejecución, siempre y cuando esto sea posible.

La problemática de este trabajo es doble, ya que por un lado hay que tener en cuenta la posibilidad de degradación de los materiales de procedencia arqueológica y por otro el conocimiento muy parcial de estas pinturas, por los pequeños restos conservados. Esto nos limitará a la hora de definir completamente la técnica de ejecución empleada ya que no se puede observar la existencia de jornadas en el caso de que las hubiera ni podemos hacer un análisis formal de los motivos decorativos.

Se han estudiado los materiales constitutivos de las diferentes capas que constituyen las pinturas, tanto pictóricas como de morteros de prepa- 
ración, así como el aspecto, textura y degradación, que presentaban los materiales.

\section{V.1. Técnicas de análisis}

- Microscopía Óptica, para el estudio de capas y la identificación de materiales tanto de la película pictórica como del mortero de base.

Las estratigrafías preparadas para su observación al microscopio óptjco se documentaron fotográficamente.

- Microscopía Electrónica de Barrido (SEM), con sistema de microanálisis por dispersión de energías de rayos $X(E D X)$, que permite un análisis elemental por capas y una imagen de la estructura material de la pintura.

- Difracción de rayos $\mathbf{X}$ nos permite identificar compuestos cristalinos, principalmente los que componen los morteros.

- Ensayos de coloración selectiva, para identificar de forma preliminar la presencia de aglutinantes orgánicos.

- Espectroscopia infrarroja por transformada de Fourier, para el análisis de las capas de preparación y de recubrimiento.

- Cromatografía de gases/espectrometría de masas, para la determinación de aglutinantes naturales, aceites resinas, ceras e hidratos de carbono (gomas, etc.).

- Cromatografía en fase líquida para el análisis de aminoácidos procedentes de las capas de temples de proteínas (sistema PICO TAG de Waters).

\section{V.2. Características de las muestras}

Las muestras se han extraído de un total de 8 fragmentos, de pequeñas dimensiones (oscilan entre los $8 \mathrm{~cm}$. de longitud máxima y $2,5 \mathrm{~cm}$. de longitud minima) y formas irregulares (son amorfos, sin aristas de terminación).

De los 8 fragmentos seleccionados 3 tienen restos de decoración, (el GUA-4112-1 y el GUA- 4111-1 y el GUA-4051-1).

En el caso del GUA-4112-1 existen tres colores, blanco, verde y rojo. De la observación visual detallada podemos deducir que la aplicación de los colores se realizó del siguiente modo: primero se aplicó el color rojo; sobre éste el verde y separando a ambos la banda blanca superpuesta a 
Estudio de materias y técnica de ejecución de los restos de pintura mural...

los dos de unos $5 \mathrm{~mm}$. de ancho. El verde no parece ser una tinta plana sino que se pueden advertir dos tonos diferentes del mismo color.

En cuanto al segundo fragmento con decoración, el n. ${ }^{\circ} 4111-1$, observamos que sobre un fondo amarillo de color plano se ha pintado una línea blanca, muy alterada, de unos $3 \mathrm{~mm}$. de ancho.

En el tercero, GUA-4051-1, se observan dos capas de pintura, una roja uniforme y otra superpuesta de color azul, en la que es difícil identificar el tipo de decoración que podía tratar ya que el fragmento es muy pequeño.

Observamos una mayor variedad de tonalidades de rojo, con el fin de poder determinar la presencia de varios pigmentos de este tipo se tomaron más muestras de los fragmentos de este color. Esta variedad no aparece en el caso de otros colores, como por ejemplo el azul, que siempre presenta características muy similares de textura, tonalidad e intensidad.

Por otra parte también se han estudiado los morteros de preparación en los fragmentos; para ello se tomaron muestras de todas las capas de preparación observables en estos morteros. No podemos determinar el número total de capas de enlucido que habia en estas pinturas, ya que ninguna de ellas estaba sobre el muro. Los fragmentos examinados a lo sumo presentaban dos capas por lo que sólo han sido éstas las estudiadas.

Como referencia indicamos las alusiones que hacen los tratadistas clásicos: Vitruvio recomienda la colocación de tres capas de enlucido a base da cal y arena, y otras tres a base de cal y polvo de mármol. Plinio por el contrario recomienda 5 capas (Vitrubio 1970: 186-195; Pline L'ancien 1985: 107).

En contraste con los autores clásicos, los resultados arrojados por estudios recientes sobre pinturas romanas concretas (Guiral, Martín-Bueno 1996: 503; Mostalac y Beltrán 1994: 41-100, García, Justo, Abad 1976: 144-152; García, Linares, Abad 1977-78: 300-301), demuestran que no siempre se aplicaba el mismo número de capas y que éste depende no sólo de la categoria del edificio sino también del tipo de estancia para la que se realizaba la pintura, y que en ningún caso eran tantas.

\section{PELICULA PICTÓRICA}

En total se tomaron 10 muestras para el estudio de las capas pictóricas. Todas se observaron mediante microscopía óptica y SEM. Además en dos de ellas se hizo un estudio completo para la determinación de aglutinantes de pintura. Los resultados del estudio de cada una de ellas, se han agrupado según la unidad estratigráfica a la que pertenecen. 


\section{VI.1. Pigmentos}

Pigmentos Unidad Estratigráfica 4111 (P1)

\begin{tabular}{|c|c|c|c|}
\hline Muestra & M. Óptica & SEM & Resultados \\
\hline HR-P1.Am. & $\begin{array}{l}\text { Capa irregular de color amari- } \\
\text { llo, tamaño de grano muy pe- } \\
\text { queño. } \\
\text { El espesor de la capa oscila } \\
\text { entre } 150 \text { y } 20 \mu \text {. }\end{array}$ & HR-P1-Am/1, Ca y Fe. & $\begin{array}{l}\text { Capa constituida por } \\
\text { Oxi-hidróxidos de hierro, li- } \\
\text { monita y goethita funda- } \\
\text { mentalmente. No se ha de- } \\
\text { tectado la presencia de } \\
\text { otros elementos como } \mathrm{Si} \text {, } \\
\text { Al, etc. que podrian indicar } \\
\text { la presencia de una tierra. } \\
\text { Es de destacar la presencia } \\
\text { de } \mathrm{Ca} \text {. }\end{array}$ \\
\hline
\end{tabular}

\begin{tabular}{|c|c|c|c|}
\hline HR-P1-A & $\begin{array}{l}\text { Granos de tamaño muy gran- } \\
\text { de y forma irregular, diámetro } \\
\text { máximo } 25 \mu \text {. } \\
\text { El espesor de la capa oscila } \\
\text { entre } 110 \text { y } 25 \mu \text {. }\end{array}$ & $\begin{array}{l}\text { Análisis de un grano HR- } \\
\mathrm{P} 1-\mathrm{A} / 1, \mathrm{Si}, \mathrm{Ca}, \mathrm{Cu} \text {. }\end{array}$ & $\begin{array}{l}\text { En la capa de pigmento } \\
\text { cuyo color es homogéneo, } \\
\text { se identifican los elementos } \\
\text { y las características típicas } \\
\text { de un vidrio. El color azul } \\
\text { se obtiene por la presencia } \\
\text { de Cu en el mismo. }\end{array}$ \\
\hline HR-P1.N. & $\begin{array}{l}\text { No se pueden observar los } \\
\text { granos de pigmento por su pe- } \\
\text { queño tamaño. El espesor de } \\
\text { la capa oscila entre } 10 \text { y } 15 \mu \text {. }\end{array}$ & $\begin{array}{l}\text { Capa orgánica, el nume- } \\
\text { ro de cuentas baja } \\
\text { mucho, solo se detecta } \\
\mathrm{Ca} \text {. }\end{array}$ & $\begin{array}{l}\text { Pigmento orgánico, proba- } \\
\text { blemente un negro de humo } \\
\text { o de carbón. }\end{array}$ \\
\hline HR-P1-R. & $\begin{array}{l}\text { Capa de espesor irregular, no } \\
\text { es continua presenta fractu- } \\
\text { ras. Se observan granos } \\
\text { grandes de color negro el es- } \\
\text { pesor de la capa oscila entre } \\
120 \text { y } 25 \mu \text {. }\end{array}$ & $\begin{array}{l}\text { HR-P1-R/1 Mg, Si, Ca, } \\
\text { Fe. }\end{array}$ & $\begin{array}{l}\text { La presencia de Ca es muy } \\
\text { importante. El pigmento } \\
\text { está constituido por una } \\
\text { tierra que tiene gran canti- } \\
\text { dad de óxidos de Fe, prin- } \\
\text { cipalmente Hematites. } \\
\text { También se pueden obser- } \\
\text { var algunos granos de } \\
\text { negro carbón. }\end{array}$ \\
\hline
\end{tabular}

\begin{tabular}{|c|c|c|c|}
\hline HR-P2-R. & $\begin{array}{l}\text { Capa roja densa y de espe- } \\
\text { sor regular, granos negros } \\
\text { bastante abundantes. } \\
\text { Oscila entre } 30 \text { y } 15 \mu \text {. }\end{array}$ & $\begin{array}{l}\mathrm{HR}-\mathrm{P} 2-\mathrm{P} / 1, \mathrm{Mg}, \mathrm{Al}, \mathrm{Si} \\
\mathrm{Ca}, \mathrm{Fe} \text {. }\end{array}$ & $\begin{array}{l}\text { Tierra roja con abundante } \\
\text { presencia de Hematites, } \\
\text { mezclada con negro de car- } \\
\text { bón. }\end{array}$ \\
\hline \multirow[t]{2}{*}{ HR-P2-B. } & 1. ${ }^{a}$ Capa roja $20 \mu$. & $\begin{array}{l}\text { 1. }{ }^{\mathrm{H}} \mathrm{HR}-\mathrm{P} 2-\mathrm{B} / 1 \\
\text { (Rojo) P, Pb, Ca, Fe. }\end{array}$ & $\begin{array}{l}\text { La capa roja está constitui- } \\
\text { da por un mezcla de tierra } \\
\text { roja y minio. }\end{array}$ \\
\hline & 2. ${ }^{a}$ Capa verde $10 \mu$ & $\begin{array}{l}\text { 2., } \mathrm{HR}-\mathrm{P} 2-\mathrm{B} / 2 \\
\mathrm{Mg}, \mathrm{Al}, \mathrm{Si}, \mathrm{K}, \mathrm{Ca}, \mathrm{Fe}\end{array}$ & $\begin{array}{l}\text { La verde extremadamente } \\
\text { fina no se ha analizado en } \\
\text { esta muestra, ya que se } \\
\text { vera en la siguiente muestra. }\end{array}$ \\
\hline
\end{tabular}


Pigmentos Unidad Estratigráfica 4112 (P2)

\begin{tabular}{llll}
\hline Muestra & M. Óptica & SEM & Resultados \\
\hline 3. ${ }^{\text {C Capa blanca } 35 \mu .}$ & $\begin{array}{l}\text { 3. }{ }^{\mathrm{a}} \mathrm{HR}-\mathrm{P} 2-\mathrm{B} / 3 \\
\text { (blanco) } \mathrm{Si}, \mathrm{P}, \mathrm{Pb}, \mathrm{Ca}, \mathrm{Fe} .\end{array}$ & $\begin{array}{l}\text { La capa blanca está } \\
\text { constituida por carbonato } \\
\text { cálcico, la presencia de } \mathrm{Pb} \\
\text { puede deberse a la mezcla } \\
\text { con blanco de plomo } \\
\text { (albayalde). }\end{array}$ \\
\hline
\end{tabular}

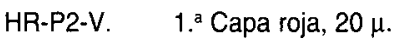

2. ${ }^{a}$ Capa verde. $10 \mu$.
HR-P2-V/1 Si, P, Pb, Ca, La capa roja esta constituida Fe.

por una mezcla de tierra roja $y$ minio.

HR-P2-V/2 Mg, Al, Si, K, La capa verde es una tierra $\mathrm{Ca}, \mathrm{Fe}$. verde, compuesta por silicatos de Fe.

HR-P2-R1. Color muy anaranjado, contorno poco definido. Entre 15 y $40 \mu$
HR-P2-R1, Mg, Al, Si, K, Pigmento muy anaranjado, $\mathrm{Ca}, \mathrm{Fe}$. constituido por oxihidróxidos de Fe, hematites, goethita y limonita.

HR-P2-R2. Capa de color muy intenso y grano muy fino. Entre $25 y$ $10 \mu$.

HR-P2-R2/1, Mg, Si, P, $\mathrm{Pb}, \mathrm{Ca}$ y $\mathrm{Fe}$
La capa roja está constituida con la mezcla de dos pigmentos, minio y tierra roja. La presencia de $P$ puede deberse a la degradación por biodeterioro.
HR-P3-A. La muestra presenta dos capas pictóricas, una primera roja, sobre la que se aplicó una capa azul de granos muy gruesos.

1. a Capa, entre 120 y $70 \mu$.

2. ${ }^{\mathrm{a}}$ Capa: entre 80 y $30 \mu$.

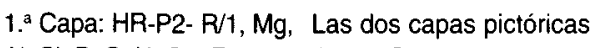
$\mathrm{Al}, \mathrm{Si}, \mathrm{P}, \mathrm{S}, \mathrm{K}, \mathrm{Ca}, \mathrm{Fe}$. tienen $\mathrm{Ca}$. La 1. ${ }^{\text {a }}$ esta compuesta por una tierra roja con importante presencia de Oxihidróxidos de $\mathrm{Fe}$, principalmente hematites. EI $P$ puede deberse a procesos de biodeterioro.

2. ${ }^{a}$ Capa: HR-P2-R/2, Al, $\mathrm{Si}, \mathrm{K}, \mathrm{Ca}, \mathrm{Cu}$. (Grano de pigmento). HR-P2-R/3 (Fondo de la La $2^{\mathrm{a}}$ es un vidrio, cuyo color se debe a la presencia de $\mathrm{Cu}$. El análisis de un grano de pigmento indica capa) $\mathrm{Cu}, \mathrm{Al}, \mathrm{Si}, \mathrm{K}, \mathrm{Ca}$, Fe. claramente que se trata de un vidrio tanto por presentarse muy oscuro en el SEM y con zonaciones, como por su composición (Si y Cu fundamentalmente). 


\section{VI.2. Aglutinantes.}

La determinación de aglutinantes orgánicos ha sido realizada por el Dr. Parra Crego de la Universidad Alfonso X el Sabio de Madrid.

Se seleccionaron dos muestras cuyas características hacian pensar en la posible presencia de este tipo de materiales y por tanto que se hubieran hecho al seco. Se tomaron de un color azul de base, muestra HR-P1-A, y de un fragmento cuyo color verde está aplicado sobre una base roja, correspondiente a una zona de decoración, muestra HR-P2-V.

A pesar de que los procedimientos e instrumentación empleados en el análisis de aglutinantes son de gran precisión y sensibilidad, no se obtuvieron resultados en la determinación de aglutinantes.

En las pruebas de tinción para proteínas las muestras estudiadas dieron positivo; sin embargo ni el análisis de infrarrojo, ni la cromatografía dieron resultados ${ }^{2}$.

Las trazas de azúcares, ácidos grasos y aminoácidos encontrados proceden probablemente de alteraciones por biodeterioro por lo que se puede descartar de forma concluyente el empleo de aglutinantes.

Por tanto podemos decir que se trata de una pintura en la que la fijación del pigmento se hace por la carbonatación de la cal, ya que ninguno de los restos orgánicos detectados se corresponden con la composición de los aglutinantes tradicionalmente utilizados en pintura mural, aún en el caso de que estos estuvieran muy degradados.

\section{VI.3. Discusión de resultados. Pigmentos y aglutinantes.}

Del estudio de las capas pictóricas examinadas, podemos deducir que los pigmentos están fijados por la acción aglomerante de la cal. Esta acción fijativa pudo deberse a la aplicación del pigmento sobre mortero fres$\mathrm{co}$, en cuyo caso tendríamos una técnica al fresco o bien pudo aplicarse mezclando el pigmento con agua de cal, en cuyo caso tendriamos una pintura "al seco", lo que llamamos un temple de cal.

\footnotetext{
2 En los ensayos de tinción se dan gran cantidad de falsos positivos en la identificación de aglutinantes cuando las tinciones se producen en capas ricas en calcita. Parra Crego, E. 1998. "Análisis químico de los materiales orgánicos en pintura murales. Aplicaciones al proceso de consolidación". Actas del Seminario Internacional sobre Técnicas de Consolidación en Pintura Mural. Pp. 14-15.
} 
Las pequeñas dimensiones de los fragmentos nos impide observar la existencia de jornadas aunque la posibilidad del empleo de una pintura al fresco es muy factible (Mora, Mora y Philippot 1984: 89-101). De ser así, pudieron aplicarse al fresco los fondos de la pintura y las grandes líneas de la composición, seguidamente el pulido de la superficie haría aflorar los restos de agua de cal y permitiría la fijación de una segunda capa de color mediante la carbonatación de esta cal.

Por otra parte estos autores señalan que los morteros romanos tenían características especiales que hacian que su fraguado fuera más lento, lo que permitiría hacer jornadas más grandes.

En cualquier caso no se puede descartar el empleo de aglutinantes en puntos muy concretos de la composición, ya que sabemos que los romanos conocian el uso de aglutinantes como la cola animal, el huevo, la goma y la leche. Además su utílización en la pintura mural hispano-romana está probada ya que en las pinturas de Bilbilis se identificó albumina de huevo en una de las muestras analizadas, aunque hay que hacer constar que su empleo debió ser mínimo ya que en este trabajo se analizaron gran cantidad de muestras y solo se detectó en una de ellas (Guiral, Martín-Bueno 1996: 524-526).

Esta hipótesis concuerda con los resultados de los análisis y con el aspecto y las alteraciones que se pueden observar en los fragmentos examinados ya que aún en los más alterados no existen escamaciones de la película pictórica en las capas de color de fondo, que sería más propio de una decoración al seco.

Los pigmentos identificados fueron de dos tipos:

\section{a) Pigmentos naturales}

Para el rojo siempre detectamos una tierra roja constituida por oxi- hidróxidos de hierro que en ocasiones se encuentra mezclada con minio, muestras HR-P2-B y HR-P2-R2. En algunos casos también se han observado granos negros de carbón que pudieron añadirse de forma intencionada para oscurecer el color de la capa pictórica, (muestras HR-P1-R y HR-P2-R). (DELAMARE 1987: 333-334; BARBET 1987: 155-161)

El pigmento blanco empleado mayoritariamente es el carbonato cálcico, probablemente aplicado en forma de $\mathrm{Ca}(\mathrm{OH})_{2}$ que por carbonatación con el $\mathrm{CO}_{2}$ de la atmósfera forma un $\mathrm{CO}_{3} \mathrm{Ca}$ que queda fijado al muro sin necesidad de aglutinante. El plomo detectado en el SEM puede atribuirse a una contaminación de la capa roja inferior; la altura del pico de $\mathrm{Pb}$ es muy pequeña y no se 
detecta de forma constante en toda la capa blanca, pero no se puede descartar el empleo de Albayalde, blanco de plomo, en la misma (Rutherford, Gettens, Kuhn, Chase 1986: 67-81; Rutherford, Gettens, West, Feller 1986: 203-207).

El verde es una tierra cuya composición química se debe a la meteorización de silicatos de calcio, magnesio y hierro (Delamare 1987: 345-373; Grisson 1986: 141-144). Autores clásicos como Vitruvio y Plinio informan sobre su utilización en la pintura mural romana (Vitruvio: (1970): 187; Pline L'ancien 1985: 57-58).

Todos los pigmentos anteriores son naturales, conocidos desde la Antigüedad y evidentemente de uso en época romana.

\section{b) Pigmentos artificiales}

Por otro lado tenemos los pigmentos artificiales como el negro y el azul, también conocidos desde la Antigüedad.

El pigmento negro obtenido a partir de la combustión de madera, lo cita Plinio al hablar del negro de vid y cuenta que se obtenía por la carbonización de pepitas de uva.

El negro de humo se conoce desde la prehistoria y se diferencia de los demás negros, por su finísimo tamaño de grano y por poseer un excelente poder cubriente y colorante, además es resistente a los ácidos y las bases. Su composición química a base de carbono impide que por el bajo peso molecular de este elemento, sea identificado en el SEM por microanálisis. Este pigmento también está debidamente documentado como propio de la paleta romana clásica (Vitruvio (1970): 190-191; Plinio 1985: 54; Harley 1982: 157-158; Doerner 1989: 59).

En cuanto al azul, se trata de un pigmento artificial obtenido por la fundición de materiales de cobre con cuarzo y álcalis. Las caracteristicas observadas tanto en microscopía óptica como en el SEM nos permiten identificarlo como tal. En el SEM los granos de pigmento se presentan de color muy oscuro y sin brillo y su composición mayoritaria es de silicio. Este elemento lo diferencia de otros posibles pigmentos de cobre. La presencia de algunos de ellos como por ejemplo azurita o malaquita, cuya identificación en el SEM se haría por la detección de $\mathrm{Cu}$, implicaría que los granos de éste fueran más brillantes y además no quedaría explicada la presencia del Si en la capa. El silicio presente en la capa pictórica forma parte de los granos del pigmento ya que en el análisis de uno de ellos los únicos elementos identificados han sido $\mathrm{Si}$, $\mathrm{Cu}$ y Ca. Esta composición coincide plenamente con el azul egipcio por 
lo que pensamos que éste fue el pigmento empleado (Uilich 1987: 323333 Mühlethaler, Thissen 1993: 113-115; Vitrubio (1970): 191; Pline L'Ancien 1985: 57).

La presencia de calcio, es constante en todas las muestras estudiadas y es atribuible a la utilización de cal para fijar la capa pictórica, ya sea por la carbonatación de un mortero fresco, ya sea por la mezcla de agua de cal con el pigmento.

\section{MORTEROS}

En total se han estudiado 10 muestras de morteros, para el estudio mediante microscopía óptica y SEM y 5 para el análisis por DRX. Las muestras corresponden a los dos niveles de mortero existentes en los fragmentos. Las hemos identificado como M1 y M2 para diferenciar los estratos existentes, siendo M1 el más profundo y M2 el más superficial. En el caso de conservarse un único nivel de mortero se ha denominado $\mathrm{M} 2$, para hacerlo corresponder con el último que constituye la base de la pintura.

En el estudio de morteros se han analizado los materiales constitutivos de los mismos, tanto áridos como aglomerantes.

La proporción de árido empleada en los morteros de Guadix, se calcula mediante el análisis semicuantitativo por DRX. A partir de estos resultados y considerando que la calcita presente en los mismos tiene su origen en el aglomerante empleado para su preparación, se puede establecer la proporción aglomerante/árido de los morteros, con un margen de error del $10 \%{ }^{3}$.

Antes de pasar a la exposición de resultados debemos hacer algunas consideraciones sobre la cuantificación de los morteros.

El estudio mediante DRX requiere eliminar la fracción de grano más grueso, que no se puede reducir a polvo en el mortero de agata. Ahora bien, puesto que las muestras corresponden a enlucidos superficiales, los de árido más fino, la fracción eliminada es relativamente pequeña.

\footnotetext{
3 El cálculo semicuantitativo de los componentes del mortero, mediante DRX, Se hace teniendo en cuenta la reflectividad de cada material, y las características de difractómetro, en este caso un Philips PW 1710 del Departamento de Mineralogía y Petrología de la Universidad de Granada. Los índices de reflectividad han sido aportados por el Dr. Francisco Huertas, de la Estación Esperimental del “Zaidín” (CSIC), Granada.
} 
En cualquier caso esta circunstancia varia algo las proporciones de los morteros y por tanto realmente estos siempre serán más ricos en árido de lo que aparece reflejado en el análisis semicuantitativo.

Por otra parte también hay que tener en cuenta que la toma de muestra está muy localizada y por tanto no es completamente representativa, por lo que las proporciones podrian variar de un punto a otro, debido a un reparto desigual de los materiales.

Por todo esto el cálculo semicuantitativo de los materiales constitutivos de los morteros no intenta ser más que una aproximación a las proporciones reales de los mismos y en ningún caso una medida exacta.

VII.1. Materiales constitutivos de los morteros de preparación

Morteros de la unidad estratigráfica, HR-4111 (P1)

\begin{tabular}{|c|c|c|c|}
\hline Muestra & M. Óptica & SEM & $D R X$ \\
\hline HR-P1-M. & $\begin{array}{l}\text { Mortero muy compacto y } \\
\text { poco poroso, el tamaño de } \\
\text { grano oscila entre } 250 \text { y } \\
10 \mu \text {, aunque puede haber } \\
\text { granos de árido menor que } \\
\text { se confundan con el } \\
\text { aglomerante. }\end{array}$ & $\begin{array}{l}\text { En todas las muestras } \\
\text { analizadas } \\
\text { correspondientes a } \\
\text { este mortero, se } \\
\text { identifican granos de } \\
\text { gran tamaño, cuyos } \\
\text { espectros son de Ca y } \\
\mathrm{Mg} \text { en unos casos y de } \\
\mathrm{Si} \text { Al y Ca en otros, } \\
\text { (calcita, dolomita y } \\
\text { cuarzo) y una matriz } \\
\text { de } \mathrm{Ca} \text {, Si y algo de } \\
\mathrm{Mg} \text {, en la que el pico } \\
\text { de Ca es muy grande. }\end{array}$ & $\begin{array}{l}\text { Sólo se pudo tomar } \\
\text { muestra de un único nivel } \\
\text { de mortero, el resultado } \\
\text { del análisis por DRX ha } \\
\text { sido muy similar en todos } \\
\text { los casos, Filosilicatos, } \\
\text { Cuarzo y Calcita. } \\
\text { Las proporciones } \\
\text { existentes en esta } \\
\text { muestra son } 56 \% \text { cuarzo, } \\
27,7 \% \text { filosilicatos y } \\
16,3 \% \text { calcita. }\end{array}$ \\
\hline
\end{tabular}


Estudio de materias y técnica de ejecución de los restos de pintura mural...

Morteros de la unidad estratigráfica, HR-4112 (P2)

\begin{tabular}{|c|c|c|c|}
\hline Muestra & M. Óptica & SEM & $D R X$ \\
\hline HR-P2-M1 & & & $\begin{array}{l}\text { Filosilicatos, cuarzo y } \\
\text { calcita. Las proporciones } \\
\text { existentes en esta } \\
\text { muestra son } 36,25 \% \\
\text { filosilicatos, } 30,11 \% \\
\text { cuarzo y } 33,46 \% \text { calcita. }\end{array}$ \\
\hline HR-P2-M2 & $\begin{array}{l}\text { Granos enormes de } \\
\text { ferrocalcitas, su diámetro } \\
\text { máximo llega hasta } 1,5 \\
\text { mm. Incluso en la parte } \\
\text { más superficial del mismo. } \\
\text { Se observan también } \\
\text { granos de cuarzo de menor } \\
\text { tamaño. }\end{array}$ & $\begin{array}{l}\text { Los granos más } \\
\text { grandes son de } \\
\text { dolomita, GC-P2-R/2, } \\
\text { Ca y Mg. } \\
\text { La matriz es de } \mathrm{Mg}, \mathrm{Al} \text {, } \\
\mathrm{Si}, \mathrm{K}, \mathrm{Ca} \text {, el pico de } \\
\mathrm{Ca} \text { es muy importante. }\end{array}$ & $\begin{array}{l}\text { Cuarzo, calcita y } \\
\text { dolomita. Las } \\
\text { proporciones existentes } \\
\text { en la muestra son } 2,51 \% \\
\text { cuarzo, } 42,84 \% \text { calcita y } \\
54,64 \% \text { dolomita. }\end{array}$ \\
\hline
\end{tabular}

Morteros de la unidad estratigráfica HR-4051 (P3)

\begin{tabular}{|c|c|c|c|}
\hline Muestra & M. Óptica & SEM & $D R X$ \\
\hline HR-P3-M 1 & & & $\begin{array}{l}\text { Filosilicatos, cuarzo y } \\
\text { calcita. Las proporciones } \\
\text { presentes en la muestra } \\
\text { son } 33,15 \% \text { filosilicatos, } \\
38,3 \% \text { cuarzo y } 28,5 \% \\
\text { calcita. }\end{array}$ \\
\hline HR-P3-M2 & $\begin{array}{l}\text { Mortero con características } \\
\text { ópticas muy similares a los } \\
\text { anteriormente vistos. }\end{array}$ & $\begin{array}{l}\text { La matriz del mortero } \\
\text { tiene una composición } \\
\text { de } \mathrm{Ca}, \mathrm{Si}, \mathrm{Mg}, \mathrm{Al}, \mathrm{P} \\
(\mathrm{SEM}, \mathrm{HR}-\mathrm{P} 3-\mathrm{A} / 4) \text {. El } \\
\text { tamaño de grano de la } \\
\text { muestra analizada en el } \\
\mathrm{SEM} \text { es muy pequeño, } \\
\text { máximo } 80 \mu \text {. }\end{array}$ & $\begin{array}{l}\text { Calcita y dolomita. Las } \\
\text { proporciones presentes } \\
\text { en esta muestra son } \\
32,45 \% \text { calcita y } 67,54 \% \\
\text { dolomita. }\end{array}$ \\
\hline
\end{tabular}

\section{VII.2. Discusión de resultados. Morteros}

Del estudio de morteros realizado, podemos extraer interesantes conclusiones, por un lado con respecto a la composición del árido y por otro con respecto a las proporciones mismo.

En general el tamaño de árido es grande, incluso en los morteros más superficiales que normalmente son los más finos. Además la composición 
de este último mortero no es exclusivamente de calcita sino mayoritariamente dolomita y cuarzo, lo que implica la utilización de arena como árido, compuesta fundamentalmente por cuarzo, filosilicatos y por dolomita. En ese caso la presencia de calcita tendría explicación en el empleo de cal como aglomerante y no en su utilización como árido.

Además las proporciones aglomerante/árido, halladas mediante el análisis por DRX, se observa que el mortero más profundo es siempre más pobre en aglomerante; las proporciones de calcita oscilan entre el 28,5 y el 33,4. Hay que hacer notar que este porcentaje correspondería aproximadamente a la relación 1 parte de cal por 2 de árido tradicionalmente utilizada.

Los morteros más superficiales aumentan su porcentaje de calcita, lo que implica una mayor riqueza de aglomerante y por tanto una mejor cohesión, las cantidades son $32 \%$ en P3 y $42 \%$ en P2. En la muestra correspondiente a P1 no se puede establecer una comparación ya que no se ha observado más que un nivel de mortero, en el que la proporción de calcita está en torno al $16 \%$, sensiblemente más baja que en los otros casos estudiados.

\section{CONCLUSIONES}

Una vez conocidos los resultados del estudio material de estas pinturas podemos llegar extraer algunas conclusiones significativas.

En lo referente a los pigmentos utilizados destacar que tanto los pigmentos naturales, como los artificiales, coinciden plenamente con la paleta que los escritos de Vitrubio y Plinio describen como la más idónea para la pintura mural:

- Amarillo, Ocrhae, a base de óxi-hidróxidos de hierro (lámina II; figuras II.4 y II.5).

- Rojos, normalmente Rubricae, a base de óxidos de hierro principalmente hematites. Además se ha constatado la presencia de un óxido de plomo que se identifica como minio, el minio de los romanos es un sulfuro de mercurio que no coincide con la composición del óxido de plomo identificado. Sin embargo Abad Casal (ABAD 1982: 395-406 atribuye esta composición al pigmento que Plinio llama minium secundarium y que se utilizaba para falsificar al primero.

Los pigmentos a base de oxi-hidróxidos de hierro son los más frecuentes, como Delamare dice constituyen un grupo muy importante por su constante utilización desde la prehistoria hasta nuestros días en todas las 
civilizaciones. Pueden encontrarse en varias formas, existiendo al menos cuatro variedades cristalinas diferentes de oxi-hidróxidos de hierro, las más importantes para pintura son la Goethita por su color ocre anaranjado y su alto índice de refracción y la variedad Hematites de color rojo. Estos materiales, muy abundantes en la naturaleza, pueden encontrarse de forma masiva o también en forma dispersa. Por otra parte es bien conocido que el calor transforma la Goethita en Hematites e igualmente que son mucho más abundantes los ocres amarillos que los rojos; por to cual el autor piensa que la fabricación de ocres rojos artificiales debía conocerse desde la Prehistoria. En el caso de las pinturas romanas, esta transformación se ha producido en ocasiones de forma accidental como consecuencia de la elevación de temperaturas producidas por incendios como por ej. en las pinturas de Herculano. A $260^{\circ} \mathrm{C}$. la Goethita vira a un rojo vivo y dependiendo de la temperatura obtendremos una variedad de tonos que está en función de su forma de cristalización, estos tonos van de un rojo intenso a un rojo oscuro, llegando incluso al negro (DELAMARE 1987: 333-335)(lámina III, fig. III.5 y III.6).

- Negro, la ausencia de un elemento característico nos hace pensar en el empleo de Atrementum, color artificial a base de negro de humo, ya que de haberse utilizado Elephantium la presencia de fósforo debería ser evidente y no es así; la combustión de marfil deja como residuo la presencia de apatito y por tanto la de fósforo.

El Attrementum según Vitrubio (Vitrubio 1970: 190) se obtenía a partir de la combustión de resinas en un horno cuya salida de humos debía estar taponada, de este modo el humo se fijaba a las paredes y se podía recoger después. Se usaba mezclado con goma como tinta para escribir y con cola para los enlucidos de las paredes. También se puede obtener este pigmento a partir de la combustión de sarmientos o teas de pino que una vez hechas brasas se apagaban y se molían en un mortero mezclados con cola, o a partir de la combustión de las "heces de vino» también molidas con cola.

- Azul, Caeruleum aegyptium es un pigmento conocido en Egipto desde la Cuarta Dinastía (2500 a. De C.) obtenido de forma artificial por el tratamiento térmico de silice, a altas temperaturas, pero en estado sólido, una sal de cobre y una sal de calcio, probablemente calcita (Ullrich 1987: 326). Con esta mezcla se hacían bolas que se exponian al fuego para obtener este pigmento. Naturalmente al ser un pigmento artificial era caro por lo que se reservaba a los lugares imprescindibles, paisajes marinos o temas mitológicos y raramente se utilizaba para fondos (Barbet 1987: 161163) (lámina II, Fig. II.1, II.2 y II.3). 


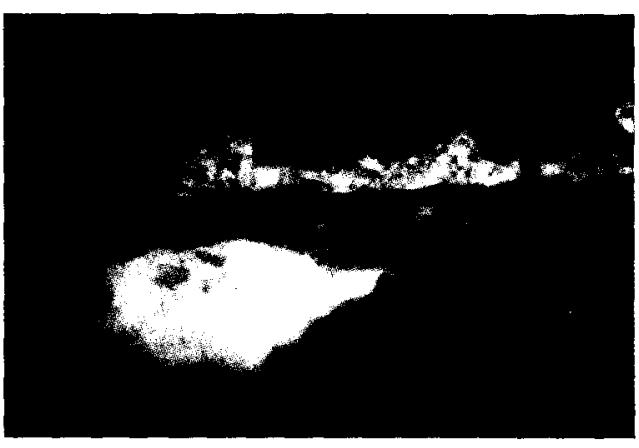

Figura II. 1

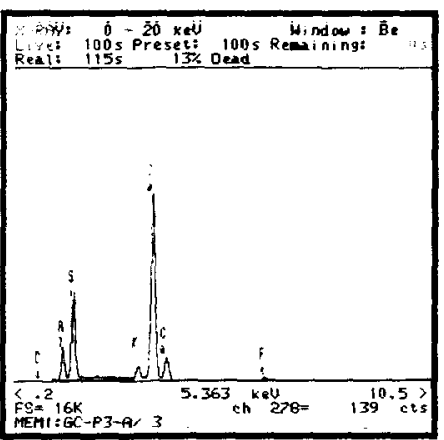

Figura II.3

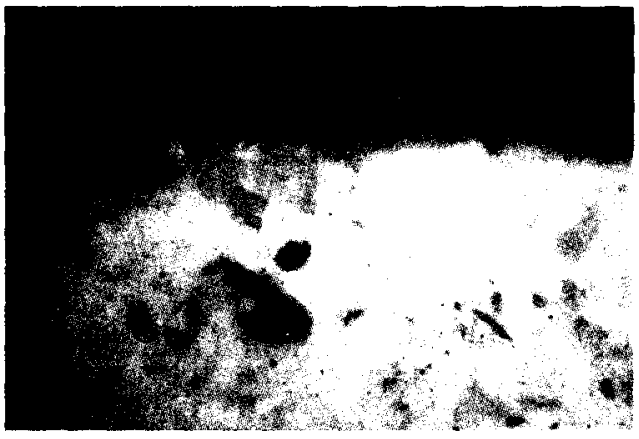

Figura II.4

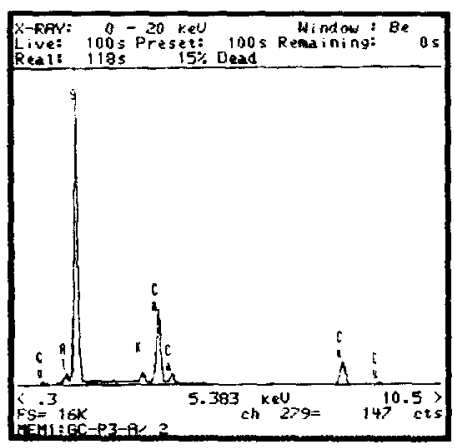

Figura II. 2

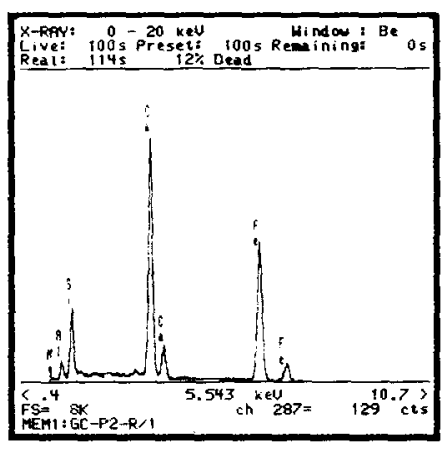

Figura II.5

Lámina II. Figura II.1. Estratigrafía de la muestra HR-P3-A. Figura II.2. SEM de la muestra HR-P3-A, correspondiente a la capa pictórica azul. Figura II.3. SEM de la muestra HR-P3-A, correspondiente a la capa pictórica roja. Figura II.4. Estratigrafia de la muestra HR-P2-R.

Figura II.5. SEM de la muestra HR-P2-R, correspondiente a la capa pictórica naranja. 
- Verde, Creta veridis, formadas por meteorización de silicatos de hierro, calcio y magnesio (Pérez-Mateos 1965: 98-99).

Del estudio que Delamare (1987: 334-337) hace sobre las tierras verdes y sobre la utilización de los verdes en general, deduce que contrariamente a lo que ocurre con los azules el empleo del verde revela particularismos locales, como por ej. la superposición de azul y amarillo para la obtención de este color en la pintura Cretense, el uso exclusivo de pigmentos verdes a base de cobre en Egipto y el uso preponderante de las tierras verdes en el mundo romano. En pintura mural se encuentra por primera vez en Pella Macedonia (siglos IV al III antes de C.), su empleo se generaliza en Roma a partir del siglo I y de allí pasa a todos las regiones del imperio: España, Francia, Inglaterra, Bélgica, Alemania, Hungría hasta Dura-Europos en Siria.

Por otra parte Plinio dice que la tierra verde es un buen sustituto de la malaquita (Pline L'ancien 1985: 57).

El microanálisis de los elementos químicos en la muestra da como resultado la presencia de hierro y el potasio como elementos característicos, la proporción de estos varía en función de la materia heterogénea que constituye la capa. En cuanto a su composición generalmente está formada por una mezcla de celadonita, glauconita y clorita. Se ha demostrado también la ausencia de cobre en este tipo de pigmento, la presencia del mismo en algunos casos debe atribuirse a las mezclas que se hacian en ocasiones con pigmentos de cobre, sobre todo con azul egipcio (Delamare 1987: 344-373) (lámina III, Fig. III.1 y III.3).

- Blanco, mayoritariamente se ha identificado cretae, carbonato cálcico. Como se ha visto en el apartado de película pictórica se identificó plomo en la capa blanca de la muestra GH-P2-B. Aunque mayoritariamente esta capa está constituida por calcita la presencia de plomo es indicativa del empleo de una mezcla de pigmentos, en este caso calcita y un pigmento blanco a base de plomo que únicamente puede atribuirse a la utilización de Cerussa, ya sea en su variante natural o en la artificial, obtenida a partir de una mezcla de plomo con vinagre, que se corresponde con el blanco de plomo actual (Rutherford, Gettens, Kühn, Chase 1993: 67-69). El albayalde se cita en todas las fuentes clásicas y es el más importante de los pigmentos blancos. De él además, se obtenia la sandáraca mediante calcinación, que según Vitrubio reunía mejores condiciones que la de origen natural (Vitrubio 1970: 191-192). Plinio sin embargo diferencia entre la sandáraca y la cerussa púrpura y opina que la mejor es la que procede de Asia (Pline L'ancien 1985: 53) (lámina III, Fig. III.1 y III.2). 
El material que fija los pigmentos es la cal, ahora bien esta cal tiene distintas posibilidades de aplicación, pudo hacerse aplicando los pigmentos sobre el mortero fresco o mezclando los pigmentos con agua de cal. Sabemos que algunos pigmentos no podían aplicarse sobre el mortero fresco, entre ellos el azul egipcio (Pline L'ancien 1985: 58 y Abad 1982:399), por lo que en este caso debería tratarse de una pintura al seco, sin embargo no se ha detectado la presencia de materia orgánica en la muestra de este color.

En cuanto al orden de aplicación de los pigmentos, según la teoría de W. LepyK Kopackzynska que adjudica el significado colores diáfanos a floridi y cubrientes a austeri, los segundos servirian de base a los prime$\operatorname{ros}^{4}$ (Abad 1982: 398-399). Podemos decir que la muestra HR-P3-A coincide con esta norma de superposición de colores: el azul egipcio, color diáfano tiene de base un rojo de Oxi-hidróxidos de hierro, color cubriente; pero en los otros casos en que existe superposición de colores se trata de pigmentos austeri, por lo que no se constata esta relación.

La comparación con otras pinturas murales analizadas en la Península, arrojan resultados muy similares. El empleo de estos materiales, no parecen únicamente consecuencia de la proximidad o abundancia de los mismos en la zona, sino más bien de una misma tradición en la decoración mural que debió extenderse por las provincias del Imperio, por tanto la riqueza de los materiales empleados dependia sobre todo de la importancia de la edificación.

Los resultados obtenidos reflejan la aplicación de los procedimientos habitualmente utilizados en este tipo de pinturas y son similares a los de otros trabajos que aunque no corresponden a esta misma zona, si son próximos cronológica y geográficamente (García, Justo, Abad 1975: 141157; GARCÍA, Linares, Abad 1977: 295-310; Mostalac, Beltrán 1994: 41125; Guiral, Martín Bueno 1996: 503-526).

En cuanto a los morteros en ningún caso llevan adiciones de yeso que puedan considerarse intencionadas. Se ha podido comprobar en el SEM que la presencia de sulfatos y cloruros es ocasional; además en el análisis por DRX no se ha identificado en ningún caso ni yeso ni cloruros, por lo que la identificación de azufre no puede atribuirse a una adición voluntaria, sino a las condiciones de conservación en que durante largo tiempo se

4 En este artículo de Abad Casal se menciona esta teoria que nos pareció interesante por coincidir con las características de superposición de colores que hemos encontrado en las pinturas de Guadix, aunque no pueda considerarse una norma definitiva. LEPYK-KOPACZYNSKA, W. "Colores floridi and austeri in der antiken Malerei". Jdl, 1958, 81 ss. 
Estudio de materias y técnica de ejecución de los restos de pintura mural...

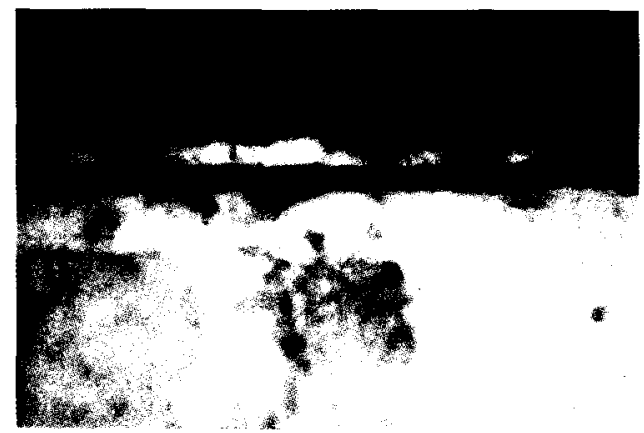

Figura III. 1

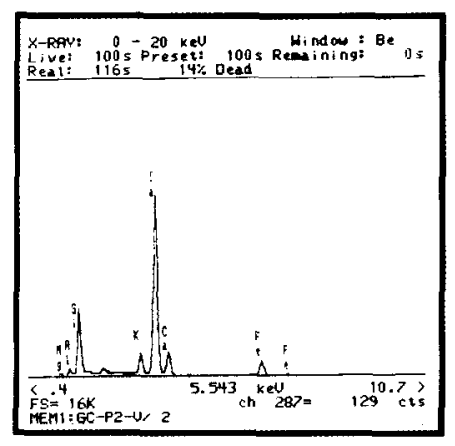

Figura III.3

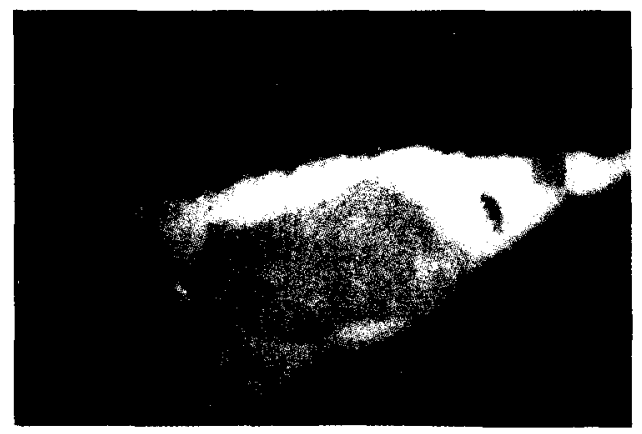

Fifura III.5

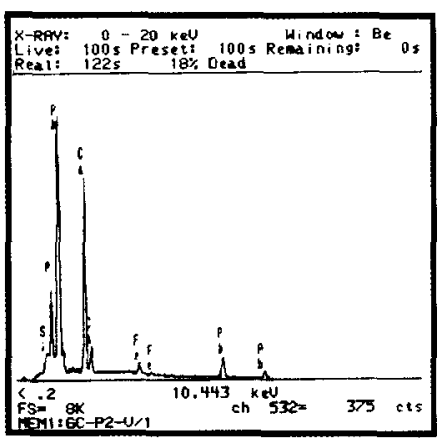

Figura III. 2

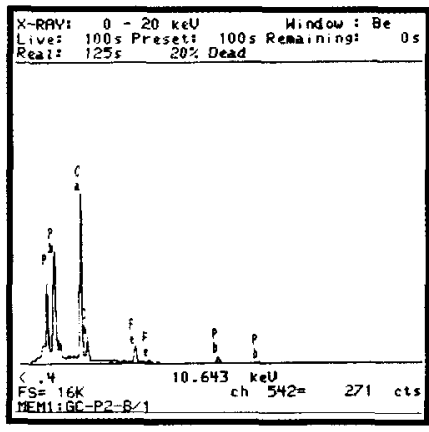

FIGURA III.4

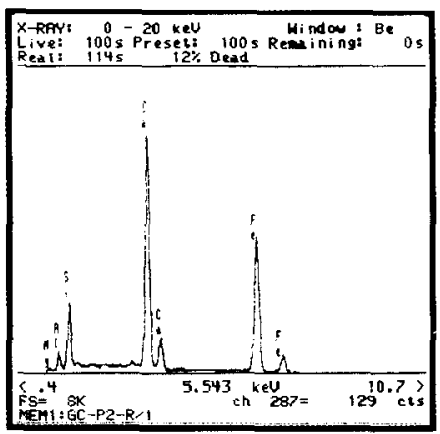

Figura III.6

Lámina III. Figura III.1. Estratigrafía de la muestra HR-P2-V. Figura III.2. SEM de la muestra HR-P2-V, correspondiente a la capa pictórica roja. Figura III.3. SEM de la muestra HR-P2-V, correspondiente a la capa pictórica verde. Figura III.4. SEM de la muestra HR-P2-V, correspondiente a la capa pictórica blanca. Figura III.5. Estratigrafía de la muestra HR-P2R1. Flgura III.6. SEM de la muestra HR-P2-R1, correspondiente a la capa pictórica rojaç 
han mantenido estos restos de pintura. Las características del material arqueológico, sometido a filtraciones de agua, que llevan en disolución sales y en los que además se producen procesos de biodeterioro, han podido producir alteraciones en las pinturas, que explican tanto la presencia de sales como la de fósforo en algunas muestras analizadas.

Aunque en un primer momento este fósforo se atribuyó a la probable presencia de un aglutinante de origen proteico, como cola o caseína, esta posibilidad fue descartada después de un exhaustivo análisis de aglutinantes de la capa pictórica de algunas muestras.

Como se dijo en el capítulo de metodología, el cálculo semicuantitativo de los materiales constitutivos de los morteros, no es más que una aproximación a las proporciones reales de los mismos y en ningún caso



Figura IV.1

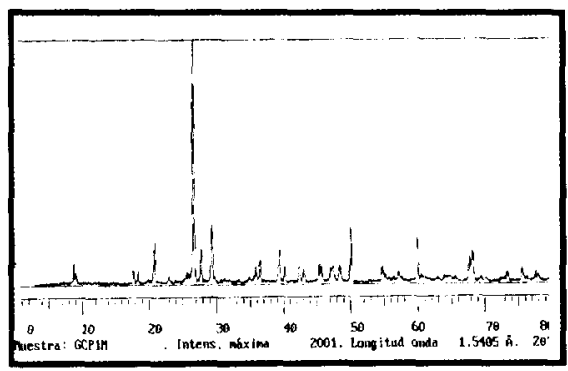

Figura IV.3

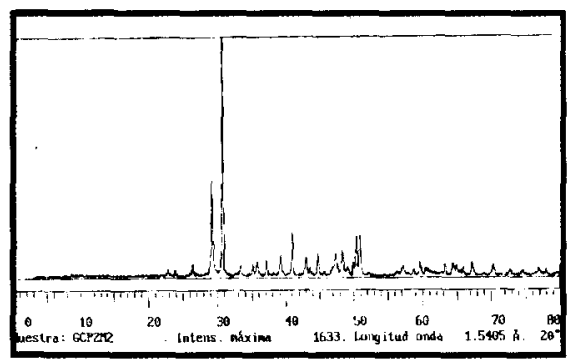

Figura IV.2

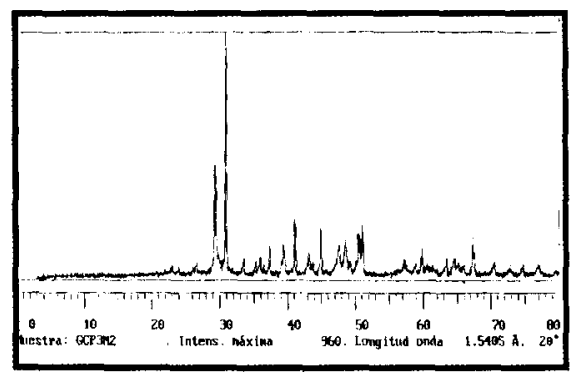

Figura IV.4

Lámina IV. Figura IV.1. DRX correspondiente al primer mortero de la muestra HR-P2. Figura IV.2. DRX correspondiente al segundo mortero de la muestra HR-P2. Figura IV.3. DRX correspondiente al mortero de la muestra HR-P1. Figura IV.4. DRX correspondiente al mortero de la muestra HR-P3. 
una medida exacta. Aun así, los resultados nos parecen interesantes sobre todo a nivel comparativo, ya que nos permiten constatar por ej. que los morteros se van haciendo más ricos en calcita a medida que son más superficiales; las proporciones del último mortero oscilan en cada caso pero siempre es más rico en calcita que el mortero que le sirve de base. Por otra parte se puede comprobar que el árido varía según los casos siendo en P3 dolomita fundamentalmente, mientras que en los otros casos es mayoritariamente de cuarzo y filosilicatos. El más pobre de los morteros superficiales es el de la unidad estratigráfica P1, que tiene alrededor de un $16 \%$ de cal, mientras que en los otros dos las proporciones coinciden, más o menos, con las tradicionalmente utilizadas. Teniendo en cuenta que se preparaban midiendo volúmenes, el porcentaje nunca será exacto. Las diferencias de proporciones entre los morteros soporte de pintura, pueden deberse a ejecuciones de distinto momento, mano de artesano o funcionalidad de la estancia (lámina IV, Fig IV.1, IV.2, IV.3 y IV.4).

Finalmente la textura lisa de algunos de los fragmentos descubiertos así como el hecho de que no se identifique aglutinante en ellos, hace pensar en la posibilidad de que estas pinturas se pulieran una vez aplicado el color. Como indicábamos en el punto VI. 3 las expolitiones permitían obtener una textura más fina y brillante, y además hacian aflorar a la superficie los restos de agua y cal que quedaban en el interior del muro y por tanto facilitaba la carbonatación de la capa pictórica. Por ello las extensiones pintadas al fresco eran mayores y se podían hacer superposiciones de capas de pintura.

Las expolitiones se usaron frecuentemente, tanto en las pinturas que imitaban mármoles como en los fondos, llegando posteriormente a generalizarse hasta que en el Cuarto Estilo dejan de utilizarse sustituyéndose por una pintura más densa en la que el empaste tiene una mayor importancia (Mora, Mora, Philippot 1984: 99-100).

\section{AGRADECIMIENTOS}

Nuestro más sincero agradecimiento al Dr. Francisco Huertas, de la Estación Experimental del Zaidín (CSIC) Granada, por la información que nos ha facilitado para el cálculo de las proporciones en los índices de reflectividad. Igualmente agradecer la colaboración del Dr. Enrique Parra Crego en el estudio de materiales orgánicos y a Dra. Carmen Guiral Pelegrín por su aportación bibliográfica. 


\section{BIBLIOGRAFIA}

Abad Casal, L. (1982): Pintura romana en España. Universidad de Alicante, Universidad de Sevilla. Tomo 1 y 2 .

- (1982): "Aspectos técnicos de la pintura mural romana", en Lucentum I, Anales de la Universidad de Alicante, Prehistoria, Arqueología e Historia Antigua.

- (1982): "Algunas consideraciones sobre los colores romanos y su emplo en pintura", en Homenaje a Saénz de Buruaga. Instituto Pedro de Valencia. Diputación Provincial de Badajoz.

ADAM, J.P. (1996): «Los revestimientos", en La construcción romana, materiales y técnicas. Ed. de los Oficios, pp. 235-249.

BARBET, A. (1987): “Qu'attendre des analyses de pigments?" en Delamare, F. Datation et caracterisation des peintures paretale et murale. PACT 17, pp. 151-170.

DelamARE, F. (1987): "Les terres vertes et leur utilisation en peinture murale romaine" en DELAMARE, F. (Eds.) Datation en caracterisation des peintures paretale et murale. PACT 17 , pp. 344-373.

- (1987): “Les pigments à base d'oxydes de fer et leur utilisation en peinture pariétale et murale". En Delamare, F. Datation et caracteristaion des peintures parétale et murale. PACT 17, pp. 333-335

DOERnER, M. (1989): Los materiales de pintura y su empleo en el arte. Ed. Reverte.

FRIzOT, M. (1980): “Études techniques des mortiers et enduits: intérêt et résultats". Peinture murale en Gaulle. Actes de séminaires 1979. Pp. 41-45.

- (1982): «L'analyse des pigments de peintures murales antiques. Etat de la question et bibliographie». Revue d'archeometrie n. ${ }^{\circ} 6$. Universidad de Rennes. Pp. 47-59.

Garcia Ramos, G. Justo Erbez, A. Abad Casal, L. (1976): «Estudio fisicoquímico y mineralógico de una serie de pinturas y revestimientos murales de Itálica (Sevilla), en Archivo Español de Arqueología, n. ${ }^{\circ}$ 48-49. Pp. 144-152.

Garcia Ramos, G. Linares López, M.D. Abad Casal, L. (1977-78): “Estudio fisicoquímico y mineralógico de algunas muestras de pinturas y revestimientos murales de Bolonia (Cádiz)" en, Archivo de Arqueologia, n. ${ }^{\circ}$ 50-51. Pp. 300-301.

Grisson, Carol A. (1986): «Green Earth», en FELLER, Robert L. (Ed.) Artists' Pigments. Vol. 1. Washington. Pp. 141-144.

Guiral Pelegrin, C. Martín-Bueno M. (1996): “Decoración pictórica y estucos ornamentales", en Bilbilis I. Institución «Fernando el Católico". Zaragoza.

HARLEY, R.D. (1982): Artistis' Pigments c. 1600-1835. Ed. Buetterworths Scientific.

Hidalgo Prieto, R. (1990): «Esquemas decorativos pictóricos de la villa romana del Ruedo (Almadinilla)". Córdoba A.A.C., 1 Pp. 109-124.

MAYER, R. (1993): Materiales y técnicas del arte. Ed. Tursen Hermann Blume.

Mora, P. Mora, L. Phillipot, P. (1984): Conservation of Wall Pintings. Ed. Butterwroths.

Mostalac Carrillo, A. Beltrán Lloris, M. (1994): Colonia Vitrix Ivlia Lepida-Celsa (Velilla de Ebro, Zaragoza). II Estratigrafia, pinturas y cornisa de la casa de los Delfines. Diputación General de Aragón. Pp. 41-357.

MühlethaleR, B. ThISsen, J. (1993): “Smalt», en Roy, A. (Ed.), Artistis’ Pigments. A Handbook of Their History and Characteristics, vol. 2. Washington. Pp. 113-130.

PLINE L'ANCIEN (1985): Histoire naturelle. Livre XXXV. Traducción y comentarios Jean Michel Croisille. Ed. Société d'Édition les Belles Lettres.

Robert L. Feller, Ed. (1986): Artists' Pigments. Vol. 1. National Gallery or Art, Washington. Oxford University Press, Oxford.

Rutherford, J. Gettens, Hermann Kühn, Chase, W.T. (1986): "Lead White», en Artists' Pigments A Handbook of Their History and Characteristics. Vol. 2. Washington. Pp. 67-99.

Rutherford, J. Gettens, West Fitzhugh, E. Fellef, R.L. (1986): "Calcium carbonate Whites", en Artistis' Pigments. Vol. 2. Washington. Pp. 203-207.

ULLRICH, D. (1987): “Egyptian Blue and Green Frit: Characterization, History and Occurrence, Synthesis". En DelamaRE, F. (Ed) Datation et caracteristaion des poeintures paretale et murale. PACT 17. Pp. 323-332.

VAQUERIzO GiL, D. et alii (1994): Arqueología cordobesa. Almedinilla. Córdoba, Seminario de Arqueología Universidad de Córdoba. Ayuntamiento de Aimedinilla.

Vitrubio, M.C. (1970): Los diez libros de la arquitectura, Ed. Obras Maestras. Barcelona, 1970. 